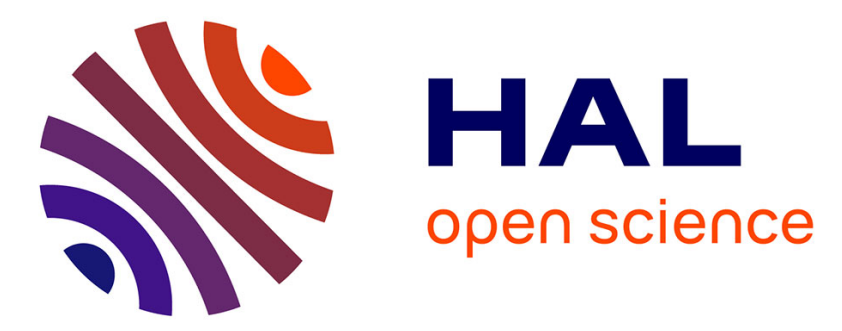

\title{
Joint processing of the parallel and crossed polarized Raman spectra and uniqueness in blind nonnegative source separation
}

Sebastian Miron, Manuel Dossot, Cédric Carteret, Samuel Margueron, David Brie

\section{To cite this version:}

Sebastian Miron, Manuel Dossot, Cédric Carteret, Samuel Margueron, David Brie. Joint processing of the parallel and crossed polarized Raman spectra and uniqueness in blind nonnegative source separation. 2010. hal-00481806

\section{HAL Id: hal-00481806 https://hal.science/hal-00481806}

Preprint submitted on 7 May 2010

HAL is a multi-disciplinary open access archive for the deposit and dissemination of scientific research documents, whether they are published or not. The documents may come from teaching and research institutions in France or abroad, or from public or private research centers.
L'archive ouverte pluridisciplinaire HAL, est destinée au dépôt et à la diffusion de documents scientifiques de niveau recherche, publiés ou non, émanant des établissements d'enseignement et de recherche français ou étrangers, des laboratoires publics ou privés. 


\title{
Joint processing of the parallel and crossed polarized Raman spectra and uniqueness in blind nonnegative source separation
}

\author{
S. Miron ${ }^{1}$, M. Dossot ${ }^{2}$, C. Carteret ${ }^{2}$, S. Margueron ${ }^{3}$, D. Brie ${ }^{1}$ \\ ${ }^{1}$ CRAN, Nancy-Université, CNRS, Boulevard des Aiguillettes, B. P. 239, \\ F-54506, Vandœuvre-lès-Nancy \\ ${ }^{2}$ LCPME, Nancy-Université, CNRS, 405 rue de Vandœuvre, F-54600, Villers- \\ lès-Nancy \\ ${ }^{3}$ LMOPS, Université Paul Verlaine - Metz, CNRS, Ile du Saulcy, B. P. \\ 80794, Metz
}

\begin{abstract}
Polarization analysis in Raman spectroscopy provides a powerful tool in chemical physics to identify the symmetry of the vibration modes of the crystals, according to their crystallographic structure and local spatial orientation. In this paper we propose two new approaches in polarized Raman spectroscopy for data with rotational and spatial diversity, respectively. We show that the joint use of parallel and crossed polarization data yields more accurate source separation results and improves the unicity properties of the solution in blind nonnegative source separation algorithms. The proposed approaches are validated on two real polarized Raman data sets.
\end{abstract}

Keywords : Raman spectroscopy, polarization, Raman imaging, nonnegative mixture identifiability, admissible solutions, uniqueness, MCR, NMF, PARAFAC

\section{Introduction}

Raman scattering is a light-matter interaction process which reflects the molecular vibration properties of the material, thus characterizing the chemical composition of the analyzed sample $[1,2]$. When coupled with a confocal microscope having motorized stages, Raman spectra can be recorded at each $(x, y)$ point on a sample grid, yielding Raman mapping [3]. For materials presenting a regular molecular structure, a more accurate characterization of the sample can be achieved by using polarizers. In particular, this is the case for crystals as their response to the polarized light excitation will reflect the crystallographic structure of the sample, motivating the development of polarized Raman spectroscopy and mapping.

In most cases the incident laser beam is linearly polarized and the Raman scattered light can be recorded in parallel or perpendicular directions by a polarized analyzer. In this paper two polarized data acquisition schemes are considered. They are respectively referred to as rotational diversity and spatial 
diversity data acquisition. The multidimensional signals recorded this way must be processed in order to get insights into the physico-chemical properties of the analyzed sample. Information about the local orientation of the sample under the microscope objective, through the retrieval of the local components of the Raman polarizability tensor [4], can thus be obtained.

Due to the multivariate nature of the data, a model which is now widely spread in spectroscopy consists in considering the data as a mixture of pure components referred to as sources, whose concentrations are varying with respect to some physical parameter referred to as diversity. The data processing consists in the retrieval of these sources and their concentrations. This problem is called multivariate curve resolution (MCR) [5] in chemometrics while in the literature of signal processing it is called nonnegative source separation or nonnegative matrix factorization [6]. The nonnegative prefix is used to stipulate the fact that source spectra and concentrations are positive by nature.

As mentioned earlier, one can record the scattered light polarized parallel or perpendicular to the incident laser polarization. Both polarizations are generally recorded but they are often processed separately. Presently, in chemical physics, for the separation of the different components from the observed mixture, the multivariate curve resolution- alternating least squares (MCR-ALS) method [5] is extensively used. SIMPLISMA -like techniques [7], based on the strong pure-variable assumption are employed to initialize the MCR-ALS algorithm. However, even if in practice the method gives generally good results, there is no guarantee that the algorithm converges to the "true" solution. In this paper we show that the joint processing of both polarizations can overcome this drawback under mild conditions and improve the estimation accuracy. We propose two signal processing models for the Raman polarized spectra acquired with angular and spatial diversity. Algorithmic solutions for the fitting of this data are given based on nonnegative matrix factorization and multilinear CANDECOMP/PARAFAC decomposition.

The rest of the paper is organized as follows : in section 2 the principles of polarized Raman data acquisition are briefly illustrated, in section 3 we present the model and the fitting algorithms for the rotational diversity polarized data and in section 4 the same thing is done for the Raman imaging data. Section 5 presents results of the proposed approaches on real spectra and some concluding remarks are given in section 6 .

\section{Polarized data acquisition}

The Raman measurements were carried out with a Jobin-Yvon spectrometer and the Raman excitation source was a Spectra-Physics ionized argon laser fixed at wavelength $\lambda=514.5 \mathrm{~nm}$. The analyzed crystal sample is fixed on a rotating stage as shown in Fig. 1. Two coordinate systems are used, one associated with the laboratory space-fixed coordinates $(0, X, Y, Z)$ and another attached to the analyzed sample $(0, x, y, z)$. The incident light is polarized such that the electric field arriving on the sample is oriented along the $Y$ direction. The scattered light is analyzed by positioning an analyzer in front of the entrance slit of the spectrometer. The analyzer is oriented either along the $Y$-axis (parallel polarization) or the $X$-axis (crossed polarization). Thus, the acquisition in one point of the sample yields a pair of spectra, one for the parallel polarization, 
indexed by $Y$ and another for the crossed polarization, indexed by $X$.

Depending on the application, two acquisition schemes can be envisaged. The rotational diversity scheme consists in rotating the sample around the $Z$ axis (Fig.1) with a fixed angular step (typically $10^{\circ}$ ) and acquiring two polarized spectra for each step of the rotation. This type of acquisition is mainly used to characterize the polarizability tensor (see. subsection 2.1) of the analyzed crystal sample system. Another acquisition scheme, used in Raman microscopy mapping, is the spatial diversity. It consists in recording polarized spectra with point-by-point scanning mode over a chosen sample area with a fixed step. This procedure permits to obtain maps of the spatial distribution of a particular component within mixture, of the analyzed sample.

\subsection{Physical considerations}

To develop the mathematical models for the polarized Raman data, it is necessary to briefly remind the physical phenomenon related to Raman scattering of polarized light. A useful quantity in this context is the polarizability tensor. The polarizability tensor is a $3 \times 3$ real-valued matrix $\boldsymbol{\alpha}$, relating the induced electric dipole moment $\mathbf{p}$ in the crystal to the electric field vector $\mathbf{e}$ of the incident light. In the crystal-fixed coordinate system $(0, x, y, z)$ the polarizability tensor can be written as:

$$
\boldsymbol{\alpha}=\left(\begin{array}{lll}
\alpha_{x x} & \alpha_{x y} & \alpha_{x z} \\
\alpha_{y x} & \alpha_{y y} & \alpha_{y z} \\
\alpha_{z x} & \alpha_{z y} & \alpha_{z z}
\end{array}\right)
$$

A Raman polarizability tensor is associated to a particular vibrational mode for a given crystal orientation [1]. The vibrational modes will be thought of as sources in the source separation problems presented in this paper. As the measurements are performed in the laboratory coordinates system $(0, X, Y, Z)$, a rotation matrix $\mathbf{R}(\phi, \theta, \chi)$ [1], accounts for the coordinate system change between the sample and the laboratory frames. Denoting the Euler angles describing the sample rotations in the coordinate system $(0, X, Y, Z)$ by $\boldsymbol{\theta} \triangleq(\phi, \theta, \chi)$, the electric vector induced in the crystal can be expressed as:

$$
\mathbf{p}(\boldsymbol{\theta})=\mathbf{R}^{T}(\boldsymbol{\theta}) \boldsymbol{\alpha} \mathbf{R}(\boldsymbol{\theta}) \mathbf{e} .
$$

If we denote by $p_{X}, p_{Y}, p_{Z}$, the three components of $\mathbf{p}$ along $X, Y$ and $Z$ axis, the intensity of the scattered light, measured by the spectrometer, is given by the following proportionality relationship:

$$
\mathbf{i}(\boldsymbol{\theta})=\left(\begin{array}{lll}
i_{X} & i_{Y} & i_{Z}
\end{array}\right)^{T} \propto\left(\begin{array}{lll}
p_{X}^{2} & p_{Y}^{2} & p_{Z}^{2}
\end{array}\right)^{T} .
$$

In practical applications only $i_{X}$ and $i_{Y}$ are measured, corresponding to the crossed and parallel polarizations, respectively.

We will suppose in the following that the recorded scattered light is a mixture of $N$ sources (vibrational modes) and $K$ wavelengths/wavenumbers are acquired for each spectrum in one point on the crystal surface. 


\section{Rotational diversity data}

In this section we focus on the angular diversity data whose model is based on the physicochemical knowledge on the analyzed crystal sample, expressed by the non-zero components of the polarizability tensor.

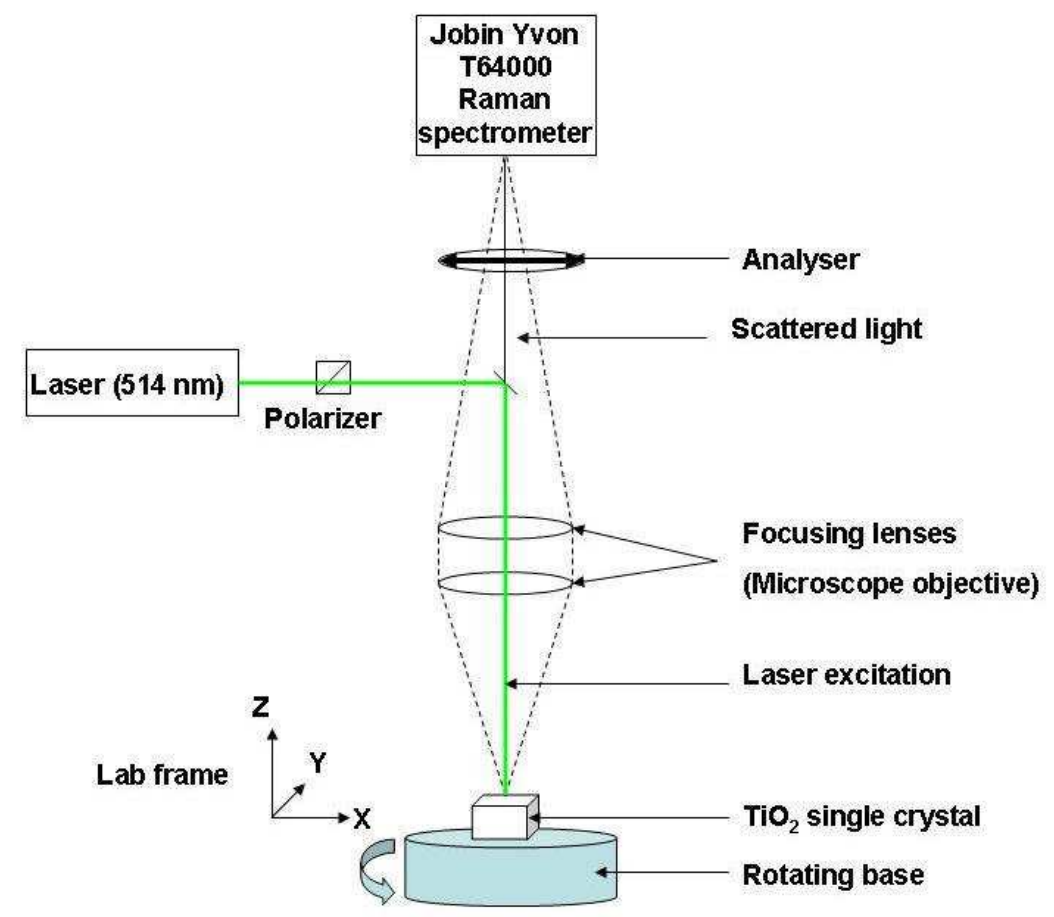

Figure 1: Polarized Raman spectroscopy principle

\subsection{The bilinear model}

For the rotational diversity acquisition scheme, $M$ polarized spectra are acquired for $M$ different rotation angles $\left(\boldsymbol{\theta}_{1}, \ldots, \boldsymbol{\theta}_{M}\right)$ of the analyzed sample. Under the generally accepted assumption of instantaneous linear mixture, the acquired data can be structured as two $M \times K$ matrices, corresponding to the two polarization orientations $X$ and $Y$ :

$$
\mathbf{D}_{p}=\mathbf{A}_{p} \mathbf{S}_{p}^{T}+\mathbf{E}_{p} \quad \text { with } \quad p=X, Y .
$$

In (4), matrix $\mathbf{E}_{p} \in \mathbb{R}^{M \times K}$ accounts for the additive noise on the sensors and the model errors and

$$
\mathbf{A}_{p}=\left(\begin{array}{ccc}
i_{1, p}\left(\boldsymbol{\theta}_{1}\right) & \cdots & i_{N, p}\left(\boldsymbol{\theta}_{1}\right) \\
\vdots & \ddots & \vdots \\
i_{1, p}\left(\boldsymbol{\theta}_{M}\right) & \cdots & i_{N, p}\left(\boldsymbol{\theta}_{M}\right)
\end{array}\right) \in \mathbb{R}^{M \times N}
$$




$$
\mathbf{S}_{p}=\left(\begin{array}{ccc}
s_{1, p}\left(\lambda_{1}\right) & \cdots & s_{N, p}\left(\lambda_{1}\right) \\
\vdots & \ddots & \vdots \\
s_{1, p}\left(\lambda_{K}\right) & \cdots & s_{N, p}\left(\lambda_{K}\right)
\end{array}\right) \in \mathbb{R}^{K \times N}
$$

are two matrices containing on their columns the angular and the spectral evolution of the scattered light for each one of the $N$ sources, respectively, and for the two polarization orientations. Thus, equation (4) illustrates two bilinear models for the two polarization types, leading a prioi to two independent source separation problems.

However, if we further analyze the underlying physico-chemical phenomenon generating the two data sets, it can be observed that the spectra of pure compounds are the same for the crossed and the parallel polarization [1,2], since the vibrational modes are imposed by the structure of the crystal. This implies $\mathbf{S}_{X}=\mathbf{S}_{Y}=\mathbf{S}$, which is quite intuitive if we consider a geometrical point of view in which the crossed and parallel polarized spectra are projections of the same signal on two orthogonal axis. By injecting this information into (4), one gets:

$$
\left(\begin{array}{c}
\mathbf{D}_{X} \\
\mathbf{D}_{Y}
\end{array}\right)=\left(\begin{array}{c}
\mathbf{A}_{X} \\
\mathbf{A}_{Y}
\end{array}\right) \mathbf{S}^{T}+\left(\begin{array}{c}
\mathbf{E}_{X} \\
\mathbf{E}_{Y}
\end{array}\right) .
$$

Eq. (7) points out a bilinear model for the polarized spectra with rotational diversity considering both polarized spectra families jointly. Besides the fact that this is a more natural and compact representation of the data, the sample size is doubled in (9) compared to (4) which should normally improve the accuracy of the estimated source parameters.

In order to simplify the presentation we use the following notations:

$$
\mathbf{D}=\left(\begin{array}{c}
\mathbf{D}_{X} \\
\mathbf{D}_{Y}
\end{array}\right), \quad \mathbf{A}=\left(\begin{array}{c}
\mathbf{A}_{X} \\
\mathbf{A}_{Y}
\end{array}\right), \quad \mathbf{E}=\left(\begin{array}{c}
\mathbf{E}_{X} \\
\mathbf{E}_{Y}
\end{array}\right) .
$$

Equation (7) can thus be re-written in a more concise manner as :

$$
\mathbf{D}=\mathbf{A} \mathbf{S}^{T}+\mathbf{E} .
$$

Given the nature of the data, the sources and the mixing coefficients are positive-valued, meaning that (9) expresses a NMF (Nonnegative Matrix Factorization) model [6].

\subsection{Identifiability of the NMF mixture model}

A source mixture model is identifiable if the sources can be uniquely estimated (up to permutation and scaling indeterminacies) from the observations. In order to discuss the the identifiability of the NMF model (9) the notion of simplicial cone needs to be introduced.

Definition 1(Simplicial cone)

The simplicial cone generated by a family of vectors $\left\{\mathbf{s}_{n}\right\}_{n=1}^{N}$ is

$$
\mathcal{C}\left(\left\{\mathbf{s}_{n}\right\}\right)=\left\{\mathbf{x}: \mathbf{x}=\sum_{n} \alpha_{n} \mathbf{s}_{n}, \alpha_{n}>0\right\} .
$$


The order of a simplicial cone is the dimension of the subspace $\operatorname{span}\left(\left\{\mathbf{s}_{n}\right\}_{n=1}^{N}\right)$.

Based on the simplicial cone, a necessary and sufficient condition for NMF identifiability has been provided by Chen in [8]:

THEOREM 1(Necessary and sufficient unicity condition)

Denoting $\mathcal{K}$ the convex hull of the data matrix $\mathbf{X}$, the decomposition of $\mathbf{X}$ according to $\mathbf{X}=\mathbf{A S}^{T}, \mathbf{A} \geq \mathbf{0}, \mathbf{S} \geq \mathbf{0}$ is unique if and only if the simplicial cone $\mathcal{C}(\mathbf{S})$, such as $\mathcal{K} \subset \mathcal{C}(\mathbf{S})$, is unique.

However, Theorem 1 does not provide any numerical conditions to determine if a NMF is unique or not. This motivated the work of $[9,10]$ from which it appears that uniqueness relies on the number of zero entries in both matrices $\mathbf{A}$ and $\mathbf{S}$. Moreover, nonnegativity alone is not enough to guarantee the identifiability of the NMF model. When uniqueness is not achieved, there is a set of possible solutions, referred to as admissible solutions. The set of admissible solutions can be determined analytically [11] for the special case of two sources while for higher size NMF it can only be solved numerically [12]. In this section we provide a geometrical interpretation of the set of admissible solutions which is used to explain why the joint use of parallel and crossed polarization data is expected to improve the NMF problem in the sense of reducing the set of admissible solutions.

For simplicity and without loss of generality, we restrain our explanations to the case of three sources, as illustrated in section 5 on real data. Consider the NMF noiseless model :

$$
\mathbf{D}=\mathbf{A} \mathbf{S}^{T} \quad \text { with } \quad \mathbf{A} \in \mathbb{R}^{M \times 3} \text { and } \mathbf{S} \in \mathbb{R}^{K \times 3} .
$$

Each raw of data matrix $\mathbf{D}$ can be seen as a point in a $K$-dimensional space. The fact that the data are nonnegative means that every such point lies in the positive orthant $\mathbb{R}_{+}^{K}$ of $\mathbb{R}^{K}$. The factorization (10) implies that all the raws of $\mathbf{D}$ lie in the simplicial cone generated by the columns of $\mathbf{S}$, namely $\mathcal{C}\left(\mathbf{s}_{1}, \mathbf{s}_{2}, \mathbf{s}_{3}\right)$. Thus, the admissible solutions for $\left(\mathbf{s}_{1}, \mathbf{s}_{2}, \mathbf{s}_{3}\right)$ are confined in the three-dimensional subspace obtained as the intersection of $\mathbb{R}_{+}^{K}$ with $\operatorname{span}(\mathbf{S})$. Let $\left\{\mathbf{e}_{1}, \mathbf{e}_{2}, \mathbf{e}_{3}\right\}$ be a canonical unitary basis of $\mathbb{R}_{+}^{K} \cap \operatorname{span}(\mathbf{S})$ (the vectors $\mathbf{e}_{1}, \mathbf{e}_{2}, \mathbf{e}_{3}$ are contained in the canonical planes of $\left.\mathbb{R}^{K}\right)$, then all the data lie inside $\mathcal{C}\left(\mathbf{s}_{1}, \mathbf{s}_{2}, \mathbf{s}_{3}\right)$ (Fig. 2).

In Fig. 3 we consider a mapping of the three-dimensional space to the plane $(1,1,1)$. By this mapping a plane in $\mathbb{R}^{3}$ is mapped to a line and a simplicial cone is mapped to a triangle. Let us suppose first that there are enough data to fully cover the triangle $\left(\mathbf{s}_{1}, \mathbf{s}_{2}, \mathbf{s}_{3}\right)$. Then any third order simplicial cone including $\mathcal{C}\left(\mathbf{s}_{1}, \mathbf{s}_{2}, \mathbf{s}_{3}\right)$ (the hatched area in Fig.3) is an admissible solution for NMF (10). This means that the problem is intrinsically ill-posed and the only sources that can be uniquely recovered are those overlapping with one of the basis vectors $\left(\mathbf{e}_{1}, \mathbf{e}_{2}, \mathbf{e}_{3}\right)$. The regularization of the problem by imposing sparseness constraints on the sources [13] reduces the solution space by dragging $\left(\mathbf{s}_{1}, \mathbf{s}_{2}, \mathbf{s}_{3}\right)$ towards the basis vectors.

Suppose now that $\mathcal{C}\left(\mathbf{s}_{1}, \mathbf{s}_{2}, \mathbf{s}_{3}\right)$ is the same as $\mathcal{C}\left(\mathbf{e}_{1}, \mathbf{e}_{2}, \mathbf{e}_{3}\right)$, meaning that the hatched area on Fig. 3 vanishes. Suppose also that the data points do not fill completely $\mathcal{C}\left(\mathbf{s}_{1}, \mathbf{s}_{2}, \mathbf{s}_{3}\right)$ (Fig. 4). Then, any third order simplicial cone con- 


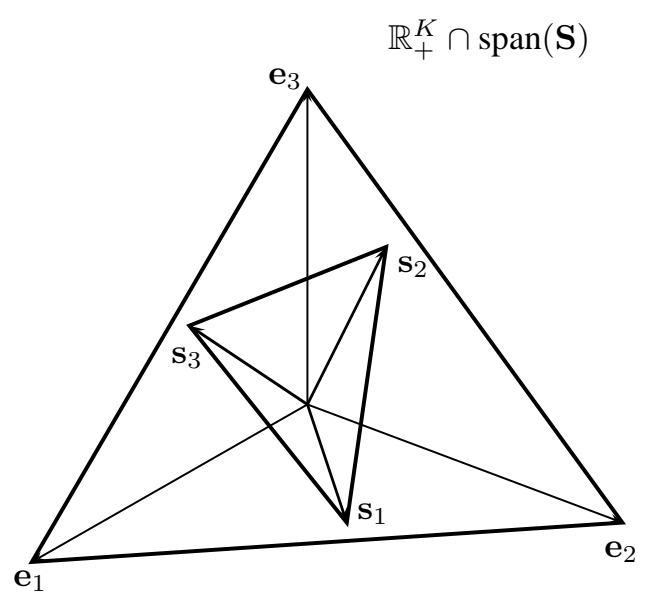

Figure 2: Geometrical illustration of $\mathbb{R}_{+}^{K} \cap \operatorname{span}(\mathbf{S})$. The vectors $\mathbf{e}_{1}, \mathbf{e}_{2}, \mathbf{e}_{3}$ form a canonical unitary basis and $\mathbf{s}_{1}, \mathbf{s}_{2}, \mathbf{s}_{3}$ are the sources.

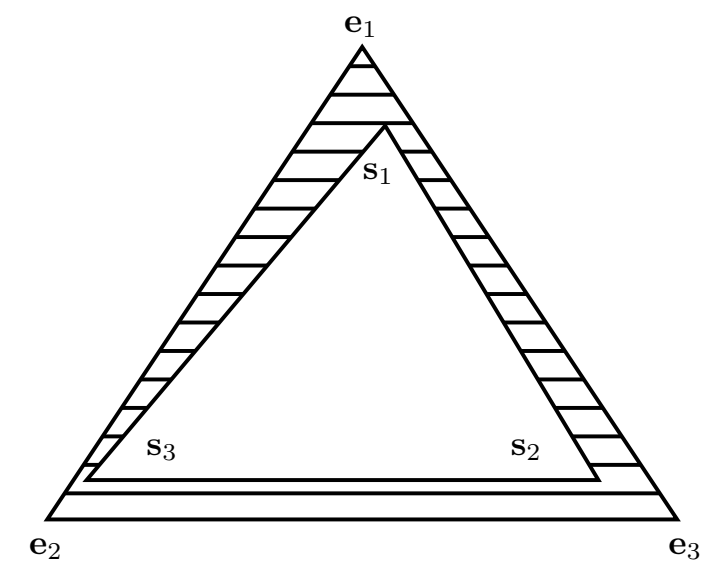

Figure 3: Mapping to the plane $(1,1,1)$ of the three-dimensional space. The hatched area corresponds to the admissible solutions domain. 


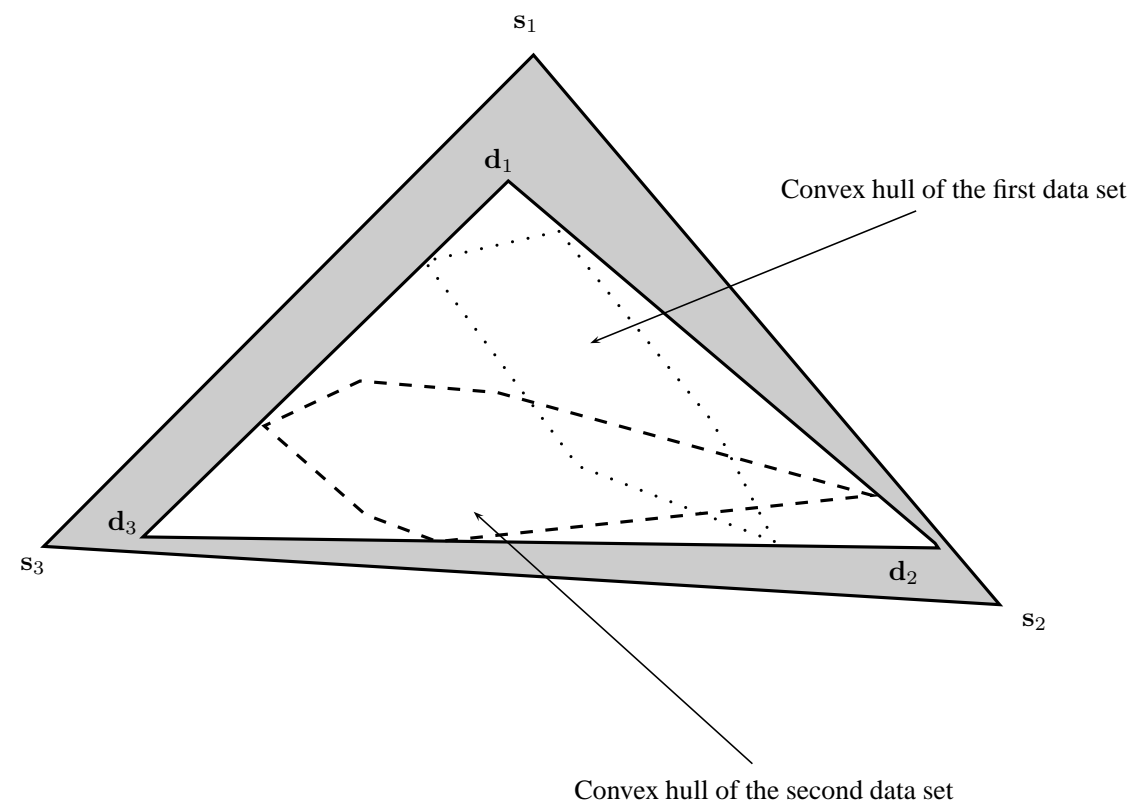

Figure 4: Illustration of the indeterminacy due to the mixing coefficients. The dotted area and the dashed area represent the crossed and parallel polarization data sets, and $\mathcal{C}\left(\mathbf{d}_{1}, \mathbf{d}_{2}, \mathbf{d}_{3}\right)$ is the simplicial cone containing the maximum number of data points on its facets.

taining all the data is an admissible solution to problem. A sufficient condition for uniqueness presented in [9] is to have at least two data points on each of the three edges of the triangle $\left(\mathbf{s}_{1}, \mathbf{s}_{2}, \mathbf{s}_{3}\right)$. In this case there is only one third order simplicial cone, namely $\mathcal{C}\left(\mathbf{s}_{1}, \mathbf{s}_{2}, \mathbf{s}_{3}\right)$, that includes all the observations. In general, the size of the admissible solutions set depends on the coverage degree of the triangle $\left(\mathbf{s}_{1}, \mathbf{s}_{2}, \mathbf{s}_{3}\right)$ by the data points. The better the data covers the triangle the smaller the solution set. This explains the improvements in the NMF admissible solutions obtained in section 5 by the joint use of crossed and parallel polarization data sets. Because different sources (vibrational modes) are characterized by different polarizability tensors it means that the sources present distinct behaviors in at least one polarized data set. Thus, it is highly probable that the observed mixtures for the crossed and parallel polarizations cover distinct areas of the simplicial cone $\mathcal{C}\left(\mathbf{s}_{1}, \mathbf{s}_{2}, \mathbf{s}_{3}\right)$. An intuitive geometrical interpretation is given in Fig. 4, where convex hulls of the two data sets (crossed and parallel) are represented by a dashed and a dotted line, respectively. It is obvious that by using both data sets jointly the incertitude on the sources (the shadowed area) is reduced compared to one set only. This result is illustrated in section 5 where NMF is applied on real data in several runs with different initial values.

By imposing sparseness on the mixing coefficients, the solution given by the smallest simplicial cone $\mathcal{C}\left(\mathbf{d}_{1}, \mathbf{d}_{2}, \mathbf{d}_{3}\right)$ is favored. Sparseness on both sources and mixing coefficients would give an intermediate soulution between $\mathcal{C}\left(\mathbf{d}_{1}, \mathbf{d}_{2}, \mathbf{d}_{3}\right)$ and $\mathcal{C}\left(\mathbf{e}_{1}, \mathbf{e}_{2}, \mathbf{e}_{3}\right)$. 


\subsection{Fitting the rotational diversity data}

For the direct model expressed by (7), the inverse problem consists in estimating $\mathbf{A}_{X}, \mathbf{A}_{Y}$ and $\mathbf{S}$ knowing $\mathbf{D}_{X}$ and $\mathbf{D}_{Y}$. Note that once the matrix $\mathbf{A}$ in (9) is estimated, it is straightforward to obtain $\mathbf{A}_{X}$ and $\mathbf{A}_{Y}$.

Alternating Least Squares (ALS) algorithms with nonnegativity constraints are generally used to estimate the matrices $\mathbf{A}$ and $\mathbf{S}$. A basic approach to invert the NMF model (9) is given in Algorithm 1. A large number of methods

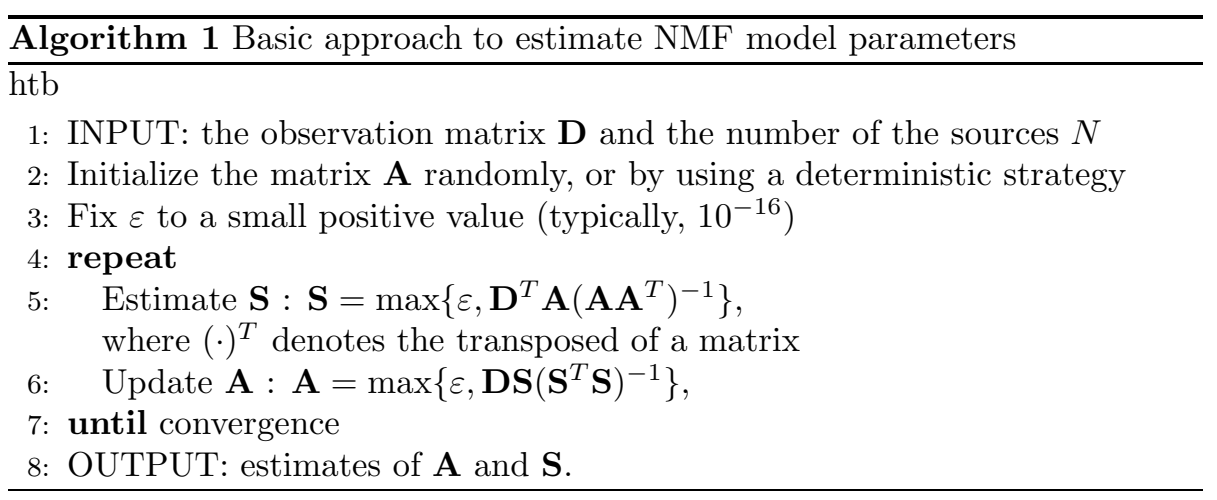

improving the performances of this basic approach have been proposed lately in the literature. One of the most popular is the NMF algorithm by Lee and Seung [14], using a multiplicative updating rule.

Also, a Bayesian Positive Source Separation (BPSS) method was developed by Moussaoui et al. [15]. The separation is performed in a Bayesian framework by encoding non-negativity through the assignment of Gamma priors on the distributions of both the source signals and the mixing coefficients. Thus, the unicity of the bilinear factorization is ensured for most practical cases, at the expense of an important computational cost. However, there is no guarantee that BPSS give the true source vectors, but only an admissible solution satisfying the Gamma priors constraints. This method will be also used in section 5 for illustration purposes.

For more details about these techniques see [16] and the references therein.

\section{Spatial diversity data}

We propose in this section a model for Raman imaging data using both crossed and parallel polarized light. The details of the Raman imaging acquisition were presented in section 2 .

\subsection{The trilinear model}

For the spatial diversity scheme, two polarized spectra are acquired in $L$ different points denoted by: $d_{1}, \ldots, d_{L}$, on a regular spatial grid. Define the following matrices:

$$
\mathbf{P}=\left(\begin{array}{ccc}
i_{1, X} & \cdots & i_{N, X} \\
i_{1, Y} & \cdots & i_{N, Y}
\end{array}\right) \in \mathbb{R}^{2 \times N}
$$




$$
\mathbf{B}=\left(\begin{array}{ccc}
b_{1}\left(d_{1}\right) & \ldots & b_{N}\left(d_{1}\right) \\
\vdots & \ddots & \vdots \\
b_{1}\left(d_{L}\right) & \ldots & b_{N}\left(d_{L}\right)
\end{array}\right) \in \mathbb{R}^{L \times N}
$$

The $\mathbf{B}$ matrix contains on its columns the spatial evolution of the source concentrations and $\mathbf{P}$ characterizes the behavior of the $N$ sources for the two polarization orientations. The acquired data can thus be expressed as two $L \times K$ matrices:

$$
\mathbf{W}_{p}=\mathbf{B} \mathcal{D}_{p}(\mathbf{P}) \mathbf{S}^{T}+\mathbf{E}_{p}, \quad p=X, Y,
$$

where $\mathcal{D}_{p}(\mathbf{P})=\operatorname{diag}\left(i_{1, p}, \ldots, i_{N, p}\right)$ is a diagonal matrix which takes the first or the second row of $\mathbf{P}$ as its diagonal, and $\mathbf{E}_{p} \in \mathbb{R}^{L \times K}$ the noise matrix. Relation (13) clearly expresses a three-way CANDECOMP/PARAFAC (CP) model [17] for the polarized data with spatial diversity.

\subsection{Identifiability issues for the trilinear model}

Before presenting the algorithms for fitting the spatial diversity data, identifiability conditions for the trilinear mixture model expressed by (13) must be discussed. The spatial acquisition scheme yields a trilinear CP model as one can see in (13). The inverse problem for this model is the estimation of $\mathbf{B}, \mathbf{S}$ and $\mathbf{P}$ from the observations $\mathbf{W}_{p}$. Unlike bilinear mixtures, the identifiability of a CP model is ensured under mild constraints given by Kruskal's condition [18]. Before stating the identifiability condition for the presented trilinear model, the Kruskal-rank of a matrix must be defined.

Definition 2(Kruskal-rank )

The Kruskal-rank of a matrix $\mathbf{B}$ equals $r\left(k_{\mathbf{B}}=r\right)$, if every $r$ columns of $\mathbf{B}$ are linearly independent but this does not hold for $r+1$ columns.

Applied to our case, Kruskal's condition states that one can uniquely estimate the matrices $\mathbf{B}, \mathbf{S}$ and $\mathbf{P}$ in (13) if:

$$
k_{\mathbf{B}}+k_{\mathbf{S}}+k_{\mathbf{P}} \geq 2(N+1)
$$

We shall only consider next, the case where $\mathbf{S}$ is a tall matrix, meaning that there are more spectral points than sources (very common in practice). Given the spiky nature of Raman spectra, $\mathbf{S}$ is full column rank with high probability, implying $k_{\mathbf{S}}=N$. Kruskal's condition can thus be re-written as:

$$
k_{\mathbf{B}}+k_{\mathbf{P}} \geq N+2 .
$$

Given the size of matrices $\mathbf{B}$ and $\mathbf{P}$, and knowing that the Kruskal-rank of a matrix is upper bounded by its classical rank, the only possible solution to inequality (15) is $k_{\mathrm{P}}=2$ and $k_{\mathrm{B}}=N$. This means that, in order to have an unique factorization, every two sources must have different polarization behaviors and $\mathbf{B}$ (with $L \geq N$ ) has to be full column rank. Thus, in practice, the CP (13) model is identifiable in most cases, provided that more acquisition points than sources are used. 


\subsection{Fitting the spatial diversity data}

Most methods for fitting the three-way CP model are derived from the Alternating Least Squares (ALS) regression which consists in estimating iteratively one matrix by fixing the two others $[17,19]$. As shown in the previous subsection this factorization is unique provided that some mild conditions are satisfied. However, the direct use of ALS can be impractical and problematic, since it usually suffers from linear convergence, and is occasionally sensitive to local minima. To avoid these problems, several algorithms have been proposed lately. In [20] an enhanced line search (ELS) is proposed to accelerate the convergence of ALS. The COMFAC algorithm introduced in [21] uses more sophisticated initializations to achieve fast, accurate convergence for factorization of trilinear arrays. Given the nature of the data, it is also natural to impose non-negativity constraints on the matrices to estimate and thereby facilitate the interpretation of the results. This can be done similarly to the bilinear case (see Algorithm 1). An optimized nonnegative algorithm for three-way factorization, used in this paper to illustrate the proposed approach, can be found in the MatLaB $N$-way toolbox developed by Bro and Anderson and freely available at: http://www.models.life.ku.dk/source/nwaytoolbox.

In order to estimate the matrices of this trilinear model one could also use one of the algorithms for nonnegative bilinear factorization mentioned in subsection 3.3 and apply it on an unfolded version of the data. This implies that, to ensure unicity on the decomposition, additional constraints on the sources and/or mixing coefficients must be made, not always physically justified. In this context, the main advantage of the trilinear algorithms over the bilinear ones is the conservation of the intrinsic structure of the data as generated by the underlying phenomena.

\section{Results}

In this section we illustrate the proposed approaches on polarized Raman spectra. The rotational diversity was explored using $\mathrm{TiO}_{2}$ rutile sample and the spatial diversity Raman spectra were collected using a mixture of $\mathrm{CaCO}_{3}$ polymorphs (aragonite and calcite).

\subsection{Results for rotational diversity data}

The approach presented in section 3 was applied to a rutile $\mathrm{TiO}_{2}$ crystal, as shown in Fig.1. The crystallographic face [001] (Hermann-Mauguin international crystallographic symbols) is analyzed. The sample is rotated with respect to $Z$ axis only, meaning $\boldsymbol{\theta}=(0,0, \chi)$. Fig. 5 presents the acquired polarized data for the parallel and crossed polarizations (matrices $\mathbf{D}_{Y}$ and $\mathbf{D}_{X}$ in (4)). The data was acquired in a spectral range of $100 \mathrm{~cm}^{-1}-800 \mathrm{~cm}^{-1}$ with an angular rotation step of 10 degrees between $0^{\circ}$ and $190^{\circ}$.

First we illustrate the effect of the joint use of the crossed and parallel polarization data sets on the size reduction of the NMF admissible solutions set, as emphasized in subsection 3.2. For the same data described earlier, the NMF algorithm [14] was used to estimate the three source vectors and the corresponding mixing coefficients. The two data sets were processed separately and jointly and the results are presented on Fig. 6 for the source spectra and on 

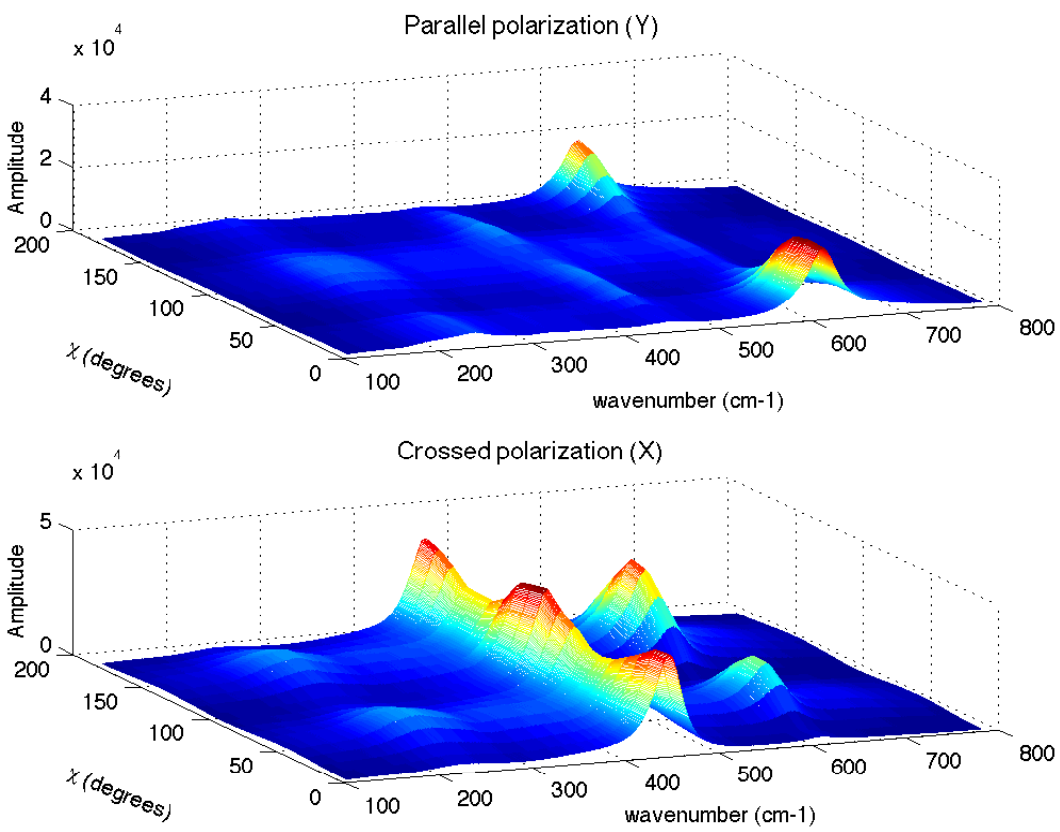

Figure 5: Polarized data for rutile $\mathrm{TiO}_{2}$

Fig. 7 and 8 for the mixing coefficients. To evaluate the size of the admissible solutions set we used 15 independent runs for each plot, with different random initial values for the matrices $\mathbf{A}$ and $\mathbf{S}$. As one can see, by processing both polarization data sets jointly (Fig. 6 (c) and Fig. 8) the admissible solution domain is largely reduced as compared to the case when only one polarization is used (Fig. 6 (a),(b) and Fig. 7). These results validate the theoretical considerations presented in subsection 3.2 and emphasize the utility of using both polarizations in Raman acquisitions.

Next, to illustrate the effect of the joint data processing on the estimation accuracy, the BPSS algorithm [15] is used, for the reasons presented in section 3.3 .

First, BPSS was applied on the concatenated data as in (7) and then on each of the polarized data sets independently, (according to eq. (4)). The results for the joint processing of the polarizations are given on Fig.9 (c) for the source spectra and on Fig.11 for the mixing coefficients. Fig.9 (a) and (b) and Fig.10 plots the estimated spectra and mixing coefficients, respectively using only the parallel or the crossed polarization data. Three sources are identified: one (\#1) with the main peak at $440 \mathrm{~cm}^{-1}$ corresponding physically to the vibrational mode $E_{g}$ (Mulliken symbols for symmetry groups [1]), another (\#2) at $610 \mathrm{~cm}^{-1}$ corresponding to the mode $A_{1 g}$ and a third one (\#3) representing, most likely, combined modes and harmonics. One can see that the use of the two polarizations jointly improves the results of the bilinear factorization. This is especially visible on the source spectra, where the polarization information allows a more accurate separation of source contributions. For example, on Fig.9 (c), source \#1 is clearly concentrated at a single wavenumber $\left(440 \mathrm{~cm}^{-1}\right)$, while 


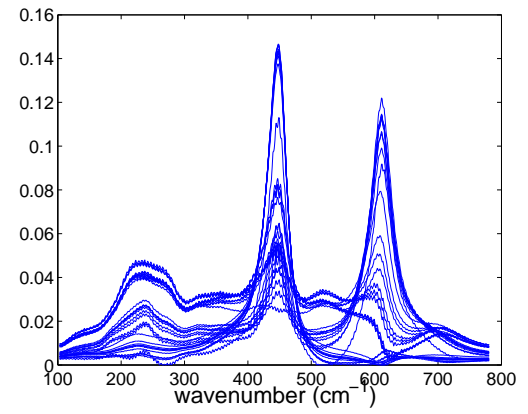

(a) Crossed polarization (X)

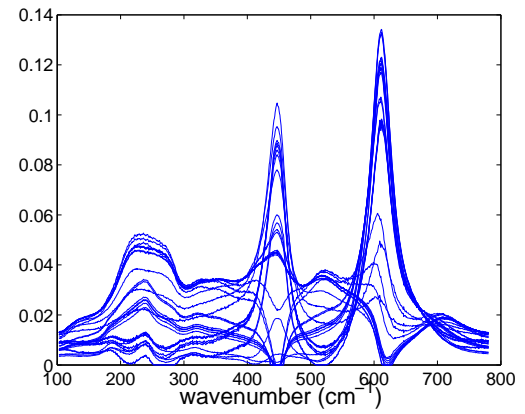

(b) Parallel polarization (Y)

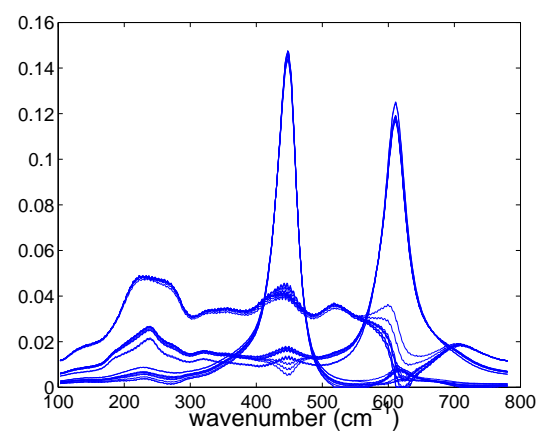

(c) Both polarization (XY)

Figure 6: Source spectra estimation by NMF (15 runs)

on Fig.9 (b) it presents an additional peak at the wavenumber corresponding to source \#2. Similar remarks can be made for the two other sources in Fig. 9 (a).

The advantage of using the polarized data jointly is also visible on the mixing coefficients. For example, if we look at the crossed polarization data in Fig. 5, one can see that the $E_{g}$ mode, i.e. source \#1, presents an offset for reasons that will be explained latter. If both datasets are used, the aforementioned characteristic is successfully recovered (Fig. 11 (a)) while this is not the case if the crossed polarization data is processed independently (Fig. 10 (a)). Similar remarks can be made for the other mixing coefficients and polarization dataset. In theory, the mixing coefficients (Fig.11) for the two data sets should pass by zero periodically, as they are theoretically proportional to $\cos ^{2} \chi$ and $\sin ^{2} \chi$, respectively. This is not the case for our data and a possible explanation to this fact is the electronic resonance phenomena which adds a baseline to the observed sources.

\subsection{Results for spatial diversity data}

For the illustration of the trilinear data model, we used a calcite crystal of several micrometers in diameter grown on an aragonite substrate, two polymorphs of $\mathrm{CaCO}_{3}$. A number of $72 \times 2$ polarized spectra was recorded on a spatial grid 


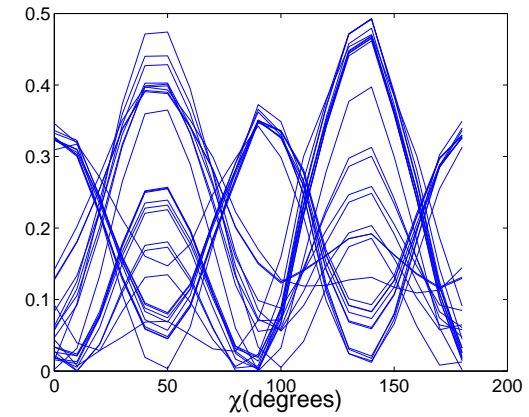

(a) Polarization X

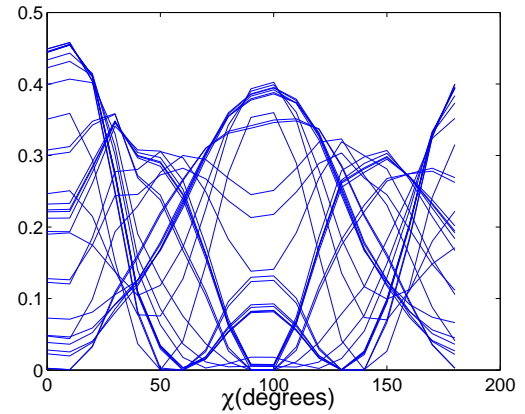

(b) Polarization Y

Figure 7: Estimated coefficients for each polarization data set separately

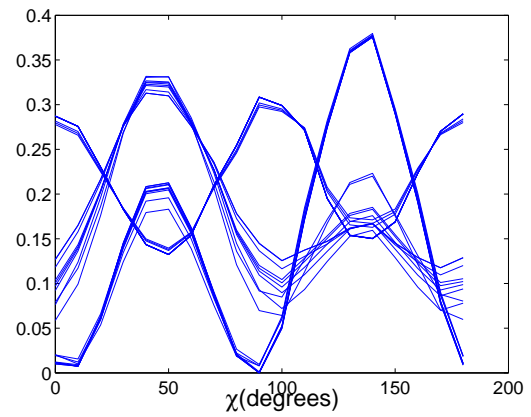

(a) Polarization $\mathrm{X}$

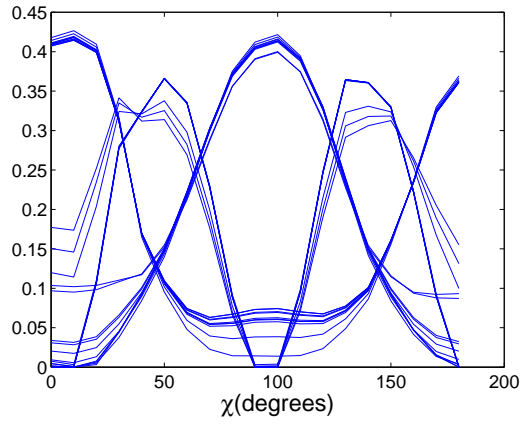

(b) Polarization Y

Figure 8: Estimated coefficients by NMF for both polarization data sets jointly (15 runs) 


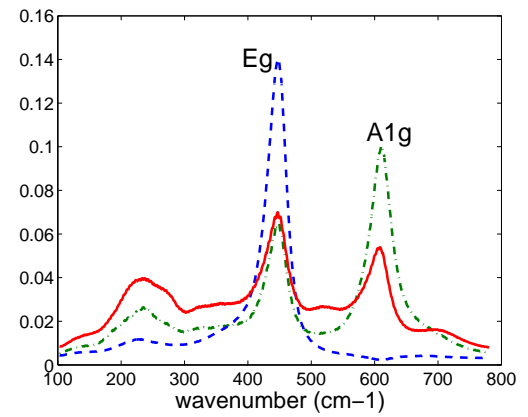

(a) Crossed polarization (X)

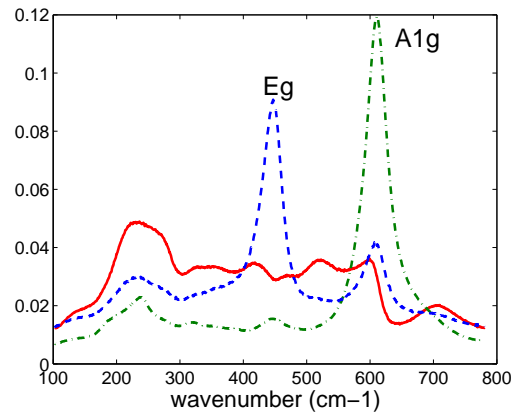

(b) Parallel polarization (Y)

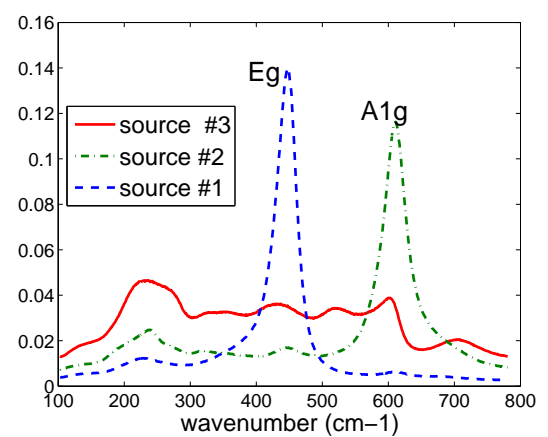

(c) Both polarization (XY)

Figure 9: Source spectra estimation

$8 \times 9$ with a spatial step of about $1 \mu \mathrm{m}$. The spectral range was $0-900 \mathrm{~cm}^{-1}$. The two families of spectra acquired in parallel and crossed polarization are presented on Fig. 12. In this case a source is no longer assimilated with one vibrational mode but rather with the totality of spectral peaks characterizing the calcite or the aragonite.

The data was processed using the PARAFAC decomposition algorithm with nonnegativity constraints described in subsection 4.3. The estimated spectral signatures and mixing coefficients for the two crystalline varieties of $\mathrm{CaCO}_{3}$ are presented on Fig.13 and Fig.14, respectively. As one can see in Fig.13 the spectral signatures of the two sources are well recovered even for the overlapping peaks around $700 \mathrm{~cm}^{-1}$. The fact that there are a lot of zero-values in the estimated spectra is due to negative values present in the analyzed data (see Fig. 12) as a side effect of the baseline removal in the preprocessing steps. The estimation of the mixing coefficients is also accurate, allowing to plot low rezolution maps of the aragonite and calcite concentrations (Fig.14 (a) and (b)), matching perfectly the optical image of the sample (not shown because of its poor graphical quality). 


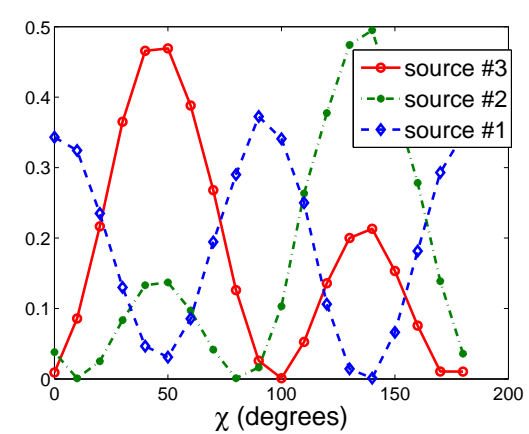

(a) Polarization X

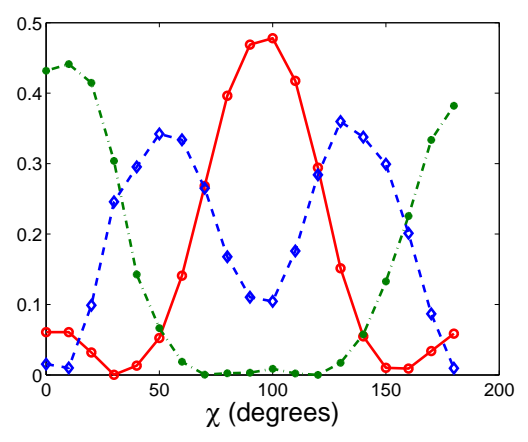

(b) Polarization Y

Figure 10: Estimated coefficients for each polarization data set separately

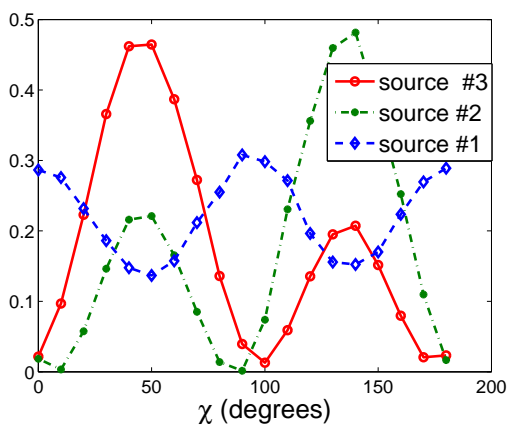

(a) Polarization X

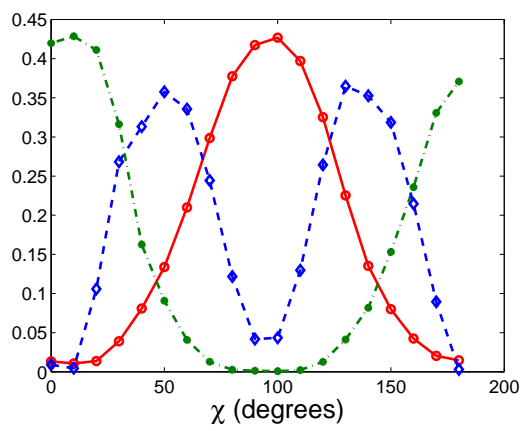

(b) Polarization Y

Figure 11: Estimated coefficients for both polarization data sets jointly

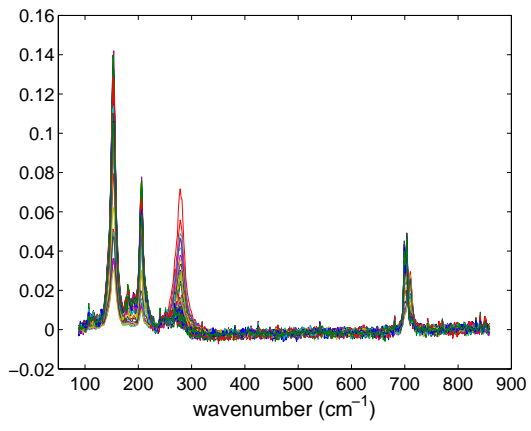

(a) Crossed polarization

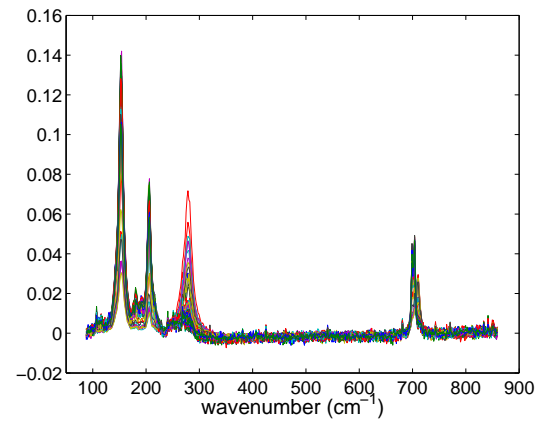

(b) Parallel polarization

Figure 12: Polarized data for $\mathrm{CaCO}_{3}$ 


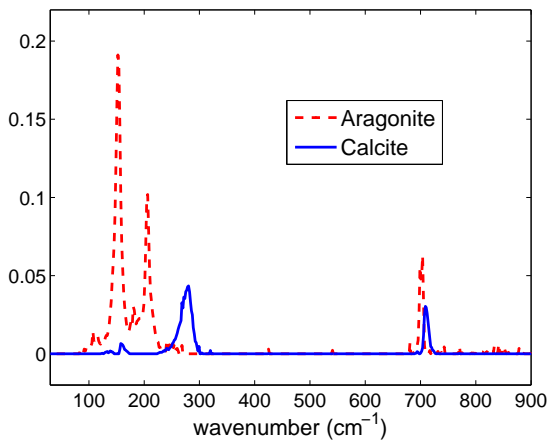

Figure 13: Spectra separation result $\mathrm{CaCO}_{3}$

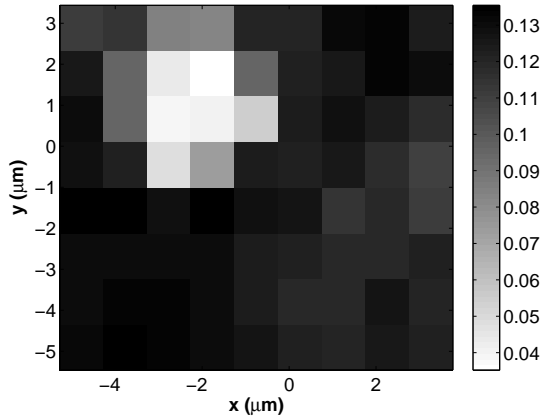

(a) Aragonite concentration map

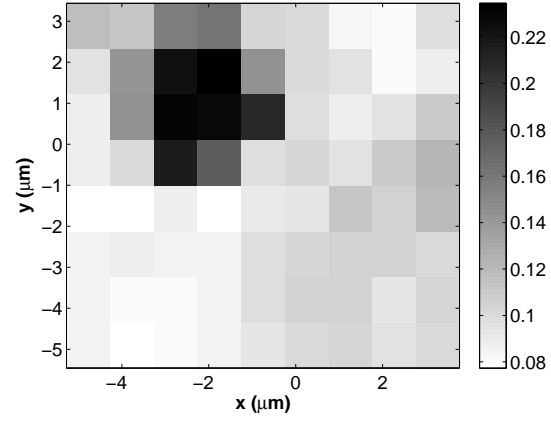

(b) Calcite concentration map

Figure 14: Concentration maps for the $\mathrm{CaCO}_{3}$ sample 


\section{Conclusions}

We proposed in this paper two approaches for dealing jointly with the crossed and parallel polarization data in Raman spectroscopy. A bilinear and a trilinear model were introduced for polarized Raman spectroscopy data with rotational and spatial diversity, respectively. We showed that, beside improving source estimation accuracy, the joint use of polarization information reduces the size of the admissible solutions set for rotational diversity data and ensures mixture identifiability in Raman imaging. The proposed approaches were validated on real $\mathrm{TiO}_{2}$ and $\mathrm{CaCO}_{3}$ spectra. These first results highlight the importance of using both crossed and parallel polarizations in Raman acquisitions and should serve as basis for future experimental protocols allowing further developments in Raman imaging and the estimation of the polarizability tensor components.

\section{References}

[1] D. A. Long, The Raman Effect: A Unified Treatment of the Theory of Raman Scattering by Molecules, John Wiley \& Sons. Ltd, 2002.

[2] G. Turrell, Infrared and raman spectra of crystals, Academic Press, 1972.

[3] S Sobanska, G. Falgayrac, J. Laureyns, and C. Brémard, "Chemistry at level of individual aerosol particle using multivariate curve resolution of confocal Raman image," Spectrochimica Acta Part A, vol. 64, pp. 11021109, 2006.

[4] Y. Batonneau, C. Brémard, J. Laureyns, J.-C. Merlin, and W. Windig, "Polarization effects of confocal raman microspectrometry of crystal powders using interactive self-modeling analysis," J. Phys. Chem. B, vol. 107, pp. 1502-1513, 2003.

[5] R. Tauler, B. Kowalski, and S. Fleming, "Multivariate curve resolution applied to spectral data from multiple runs of an industrial process," $A n$ alytical chemistry, vol. 65, no. 15, pp. 2040-2047, 1993.

[6] P. Paatero and U. Tapper, "Positive matrix factorization : A nonnegative factor model with optimal utilization of error estimates of data values," Environmetrics, vol. 5, pp. 111 - 126, 1994.

[7] W. Windig and J. Guilment, "Interactive self-modeling mixture analysis," Analytical chemistry, vol. 63, no. 14, pp. 1425-1432, 1991.

[8] J.C. Chen, "The nonnegative rank factorizations of nonnegative matrices," Linear algebra and its applications, vol. 62, pp. 207-217, 1984.

[9] D. Donoho and V. Stodden, "When does non-negative matrix factorization give correct decomposition into parts?," in Advances in Neural Information Processing, 2003, vol. 16.

[10] H. Laurberg, M. G. Christensen, M. D. Plumbley, K. L. Hansen, and S. H. Jensen, "Theorems on positive data: On the uniqueness of NMF," Computational Intelligence and Neuroscience, vol. 2008, no. 764206, 2008. 
[11] S. Moussaoui, D. Brie, and J. Idier, "Non-negative source separation: range of admissible solutions and conditions for the uniqueness of the solution," in Proc. IEEE International Conference on Acoustics, Speech and Signal Processing, Philadelphia, USA, March 2005.

[12] S. Moussaoui, D. Brie, C. Carteret, and A. Mohammad-Djafari, "Bayesian analysis of spectral mixture data using Markov Chain Monte Carlo methods," Chemometrics and Intelligent Laboratory Systems, pp. 137-148, 2006.

[13] P. O. Hoyer, "Non-negative matrix factorization with sparsness constraints," Journal of Machine Learning Research, vol. 5, pp. 1457-1469, 2004.

[14] D. D. Lee and H. S. Seung, "Learning the parts of objects by non-negative matrix factorization," Nature, vol. 401, no. 6755, pp. 788-791, 1999.

[15] S. Moussaoui, D. Brie, A. Mohammad-Djafari, and C. Carteret, "Separation of non-negative mixture of non-negative sources using a Bayesian approach and MCMC sampling," IEEE Trans. Signal Process., vol. 54, no. 11, pp. 4133-4145, nov 2006.

[16] A. Cichocki, R. Zdunek, and S. Amari, "Nonnegative matrix and tensor factorization," IEEE Signal Processing Magazine, pp. 142-145, Jan. 2008.

[17] R.A. Harshman, "Foundations of the PARAFAC procedure: Model and conditions for an 'explanatory' multi-mode factor analysis," UCLA Working Papers Phonetics, vol. 16, pp. 1-84, Dec. 1970.

[18] J.B. Kruskal, "Three-way arrays: Rank and uniqueness of trilinear decompositions with application to arithmetic complexity and statistics," Linear Algebra Applicat., vol. 18, pp. 95-138, 1977.

[19] A. Smilde, R. Bro, and P. Gheladi, Multi-Way Analysis. Applications in the Chemical Sciences, Wiley, Chichester, U.K., 2004.

[20] M. Rajih and P. Comon, "Enhanced line search: A novel method to accelerate PARAFAC," SIAM Journal on Matrix Analysis and Applications, vol. 30, no. 3, pp. $1128-1147,2008$.

[21] R. Bro, N.D. Sidiropoulos, and G.B. Giannakis, "A fast least squares algorithm for separating trilinear mixtures," in Proc. Int. Workshop Independent Component Analysis and Blind Signal Separation (ICA), Aussois, France, Jan. 1999. 